\title{
Galileo's Refutation of the Speed-Distance Law of Fall Rehabilitated $^{*}$
}

John D. Norton

Center for Philosophy of Science

Department of History and Philosophy of

Science

University of Pittsburgh

http://www.pitt.edu/ jdnorton
Bryan W. Roberts

Department of History and Philosophy of

Science

University of Pittsburgh

Note to typesetter: Please use attached high-res EPS images in place of the low-res images embedded in the text.

\begin{abstract}
.
Galileo's refutation of the speed-distance law of fall in his Two New Sciences is routinely dismissed as a moment of confused argumentation. We urge that Galileo's argument correctly identified why the speed-distance law is untenable, failing only in its very last step. Using an ingenious combination of scaling and self-similarity arguments, Galileo found correctly that bodies, falling from rest according to this law, fall all distances in equal times. What he failed to recognize in the last step is that this time is infinite, the result of an exponential dependence of distance on time. Instead, Galileo conflated it with the other motion that satisfies this 'equal time' property, instantaneous motion.
\end{abstract}

Keywords: acceleration, freefall, foundations of physics, Galileo, history of physics

\section{Introduction}

Our concern is a single paragraph in Galileo's Two New Sciences, in which Galileo purports to refute the law of fall that sets speed proportional to distance fallen. After Sagredo admits to finding this speed-distance law reasonable, Salviati responds with some unusual consolation, revealing that 'your reasoning has in it so much of the plausible and probable, that our Author himself did not deny... that he had labored for some time under the same fallacy.' Salviati now promises to dispose of the speed-distance law 'as false and

\footnotetext{
* We are grateful to [NAMES REMOVED FOR BLIND REFEREEING] for helpful remarks on an earlier
} draft. 
impossible as [it is] that motion should be made instantaneously, and here is very clear proof of it.' He then presents the following now-infamous argument.

When speeds have the same ratio as the spaces passed or to be passed, those spaces come to be passed in equal times; if therefore the speeds with which the falling body passed the space of four braccia were the doubles of the speeds with which it passed the first two braccia, as one space is double the other space, then the times of those passages are equal; but for the same moveable to pass the four braccia and the two in the same time cannot take place except in instantaneous motion. But we see that the falling heavy body makes its motion in time, and passes the two braccia in less [time] than the four; therefore it is false that its speed increases as the space. (Galilei, 1974, pp. 160.)

This is an important moment in the dialogue. The path to one of the main results of the work, the final law of fall, is impeded by the speed-distance law of fall. In his own investigations Galileo needed to pass beyond it. Presumbaly it was a significant obstacle, since it is one of the two instances in the Two New Sciences in which an error or confusion by the Author himself is reported. ${ }^{1}$

The proof or demonstration (dimostrazione) is designed to enable the reader to pass beyond this erronous view. We do not know if this is the proof that convinced Galileo of the inadmissibility of the speed-distance law in his own development; all we know of his biography is that he did reject it. Nonetheless, it is the only known text, published or otherwise, in which Galileo discusses the rejection of the speed-distance law. So, it is an important passage that we should make special efforts to understand. Hence, it is a matter of some awkwardness that later commentators to the present day have found Galileo's text puzzling, and have strained to discern how Galileo's argument was intended to proceed. These efforts often collapsed into the accusation that Galileo was guilty of a transparent fallacy.

1 The other instance occurs in Day 4, when Galileo admits to having 'long remained in the same shadows' in trying to understand the nature of impact. 
We shall urge here that there is a reading of Galileo's argument in which it does precisely what he suggests and does it quite cogently. In particular, we hold that Galileo's argument passes through the following three steps.

1. Scaling. Galileo states a general result of the equal time of passage for two motions when one is produced by scaling the other.

2. Self Similarity. Galileo notes that the scaling appears as a self-similarity within the one motion governed by the speed distance law of fall; and that this entails that for it, all spaces are covered in the same time.

3. Downfall. Galileo concludes that this condition is compatible only with instantaneous motion. The sole error of the analysis enters here in that Galileo overlooked, as other commentators as far back as Mach have remarked, that this equal time condition is compatible with an infinite time of fall as well.

That Galileo's first step asserts a scaling relation between two motions is where our analysis diverges from earlier treatments. Although the importance of translating Galileo's velocità as in the plural as speeds has been recognized after Drake (1970, pp. 21-43), these speeds are usually assumed to pertain to the same motion. On that reading, Galileo's first premise is little different from his conclusion, in which case he has no 'proof' at all, much less a very clear one. In contrast, our analysis develops out of the idea that the speeds pertain to a pair of distinct motions. It is this difference of starting point that allows us to read Galileo's text as presenting a cogent argument.

\section{Confusions over Galileo's Refutation}

It's worth briefly reflecting on the three-and-a-half centuries of consternation caused by the challenge of interpreting this passage. Galileo's critics rejected it outright, along with most of his other discussions of freefall. Pierre le Cazré even suggested that Galileo erroneously applied a rule of uniform speed to accelerated motion. ${ }^{2}$ Galileo's supporters found his argument just as puzzling. Marin Mersenne thought that the law

2 For a discussion of Galileo’s early critics, see (Galluzzi, 2001). 
Galileo claimed to refute 'can nevertheless be understood in a correct way' (Mersenne, [1639] 1973a, pp. 184, our translation). Even the careful Pierre de Fermat suggested that, if this passage really did hint at a precise demonstration, then Galileo 'saw or believed himself to see the demonstration in obscurity' (Fermat, [1646?] 1894, vol.2, 268, our translation). ${ }^{3}$ At least Tenneur (1649) seemed to recognize one significant part of Galileo's argument, which involves a technique we call self-similarity. But in order to recover Galileo's conclusion, Tenneur assumed that Galileo was implicitly reasoning about average velocities, a claim that is poorly supported by Galileo's text. ${ }^{4}$

Modern commentators have not fared much better than Galileo's contemporaries. Ernst Mach renewed scholarly interest in Galileo's argument, but called it 'a course of fallacious reasoning' (Mach, 1919, pp. 247). In particular, Mach thought that Galileo's result disagreed with modern classical mechanics, suggesting that the law Galileo purported to refute is not inherently absurd, but simply does not accord with experience (Mach, 1919, pp. 248). Damerow et al. have recently echoed this sentiment. ${ }^{5}$

Cohen and Hall each later suggested Galileo's passage could be correctly understood as a clever one-line argument, as long as we take Galileo to use the mean-speed theorem implicitly. ${ }^{6}$ This interpretation was convincingly refuted by Drake (1970), who showed that it was based on an incorrect translation, and argued that the mean-speed theorem played no role in Galileo's text, or in his unpublished manuscripts on freefall.

Later commentators seem to have recycled many of the mistakes of Galileo's

3 See Roberts (forthcoming) for an exposition of Fermat's reconstruction of Galileo.

4 Palmieri has pointed out that Galileo's celebrated student, Evangelista Torricelli, worked on a structure whose properties are analogous to the motions conforming to the speed-distance law. In a geometric spiral, the radial distance of the curve from the origin grows geometrically with each angular cycle completed. Taking each angular cycle to correspond to a unit of time elapsed, this radial component turns out to implement the motion prescribed by the speed-distance law. Torricelli's demonstration of the possibility of the geometric spiral amounted to an indirect repudiation of Galileo's argument against the speed distance law and, Palmieri suggests, this fact may have played a role in Torricelli's treatment of the spiral. See (Palmieri, 2009, pp. 131-142).

5 They write: 'Galileo's argument is also incompatible with classical mechanics, since, in classical mechanics, it does not follow that a motion whose velocity increases in proportion to the distance traversed must be instantaneous but rather that such a motion cannot begin at all from the state of rest' (Damerow et al., 1992, pp. 236).

6 Also called the 'Merton rule,' the mean speed theorem says that the average speed of a uniformly accelerated body is equal to the average of its initial and its final speeds. See (Cohen, 1956, pp. 231-235) and (Hall, 1958, pp. 342-349). 
contemporaries. Drake thought that Tenneur had already 'understood Galileo's reasoning exactly' (1970, pp. 35). However, the reasoning Drake reconstructed from Tenneur is not enough to recover Galileo's conclusion ${ }^{7}$. Recent Mersenne commentators later sided with Cazré, suggesting that Galileo's argument was either a 'slight of pen,' or else that Galileo was 'applying the law of uniform motion to a motion which is not so' (Mersenne, 1973b, pp. 250, our translation).

Most recently, Damerow et al. argue that Galileo had applied a rule of 'proportionality between distance and velocity in the sense of the Aristotelian concept' (Damerow et al., 1992, pp. 235), referring to a concept of velocity they take Galileo to have evidenced in a famous 1604 letter to Sarpi ${ }^{8}$ They conclude that Galileo's argument does not really work, and that this provides evidence for the incompatibility of Galileo's mechanics with classical mechanics:

The refutation of space proportionality in the Discorsi is thus no refutation of these proofs but of the proportionality between the degree of velocity and distance under the presupposition of a proportionality between overall velocity and degree of velocity. But Galileo's argument is also incompatible with classical mechanics... (Damerow et al. 1992, 236.)

Let us now see how these difficulties can be avoided.

\section{Galileo's Argument}

Our contention is that Galileo's refutation works almost exactly as he said and was very much less confused than later commentators have suggested. More precisely, Galileo's argument may be divided into three steps. The first two work exactly as Galileo intended and are by themselves sufficient to demonstrate the untenability of the speeddistance law for ordinary fall. The third step almost works; it is defeated at the last moment

7 Drake interpreted Galileo: 'If each conceivable velocity passed through in the whole descent is the double of a velocity passed through in the first half of the descent, then there is no way of accounting for a difference in the time required for one descent as against the other. That is all there is to his argument' (Drake, 1970, pp. 33). But of course, Galileo's conclusion does not follow from this alone.

8 The argument Galileo develops in this letter, although interesting, will play no role in the interpretation we present. For a discussion, see (Drake, 1969) and (Damerow et al., 1992). 
by a natural, but false assumption. The three steps are described below.

\subsection{Scaling}

Galileo begins the argument by asserting:

When speeds have the same ratio as the spaces passed or to be passed, those spaces come to be passed in equal times (Galilei, 1974, pp. 160).

Here Galileo is noting a scaling property of speed and distance. Start with a motion described by fixing which speed a body has at each point of the space traversed. We can scale up the motion by increasing the speed in the same proportion as the space. This results in a new motion in general distinct from the one we started with. For example, we might double the space traversed and also double the corresponding speeds at each position to create a new motion. The scaling result asserts that the time required by the scaled up motion to traverse the new, scaled up space is the same as is required by the original motion to traverse the original, unscaled space with the unscaled speeds. See Figure 1 for a representation of the scaling.

This scaling result is a very general result. It holds, obviously, for a constant speed. It is not hard to convince yourself that it also holds for variable speeds, no matter how the speed may vary with the space. 


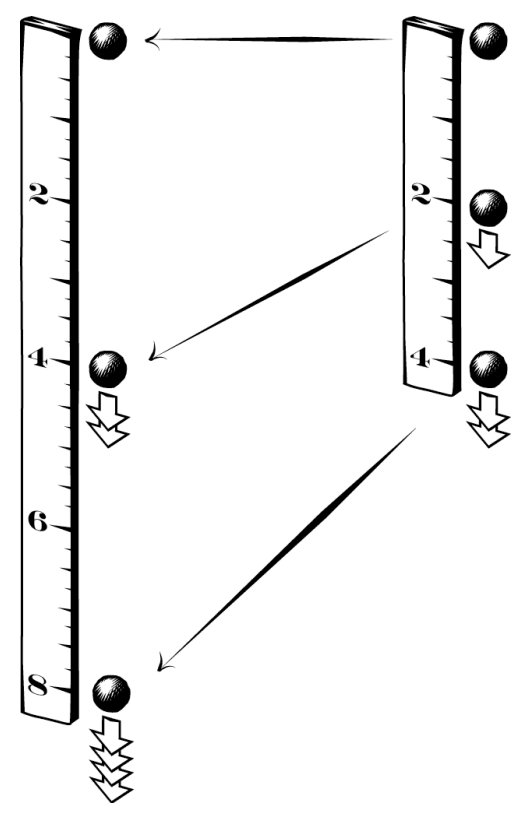

Figure 1. Scaling a motion.

\subsection{Self Similarity}

Galileo continues:

...if therefore the speeds with which the falling body passed the space of four braccia were the doubles of the speeds with which it passed the first two braccia, as one space is double the other space, then the times of those passages are equal

Galileo is now considering a result that holds just for the particular case of a law of fall in which the speed is proportional to the space fallen. This motion has the property of selfsimilarity. Take that portion of the motion that covers the first four feet fallen and scale it up by doubling. The result is not a new motion but merely the same motion now extending to eight feet. That is, the whole is a scaled-up version of the part. Figure 2 shows how the original motion is contained within the scaled-up motion as a part.

Galileo can now apply the earlier scaling result to infer to the 'Equal Time' result:

(ET) The time taken to cover the first four feet is the same as the time required to cover the full eight feet. 
So far, Galileo's reasoning has been flawless. He has correctly discerned the properties of the speed-distance law of fall, where by 'correctly' we merely mean that he has found properties logically entailed by the law and discernible with techniques routinely used by him. Moreover, the result (ET) already establishes that the law is not a viable candidate as the law governing the fall of bodies of ordinary experience. For we know from ordinary experience that bodies take longer to fall eight feet than they do to fall four feet.

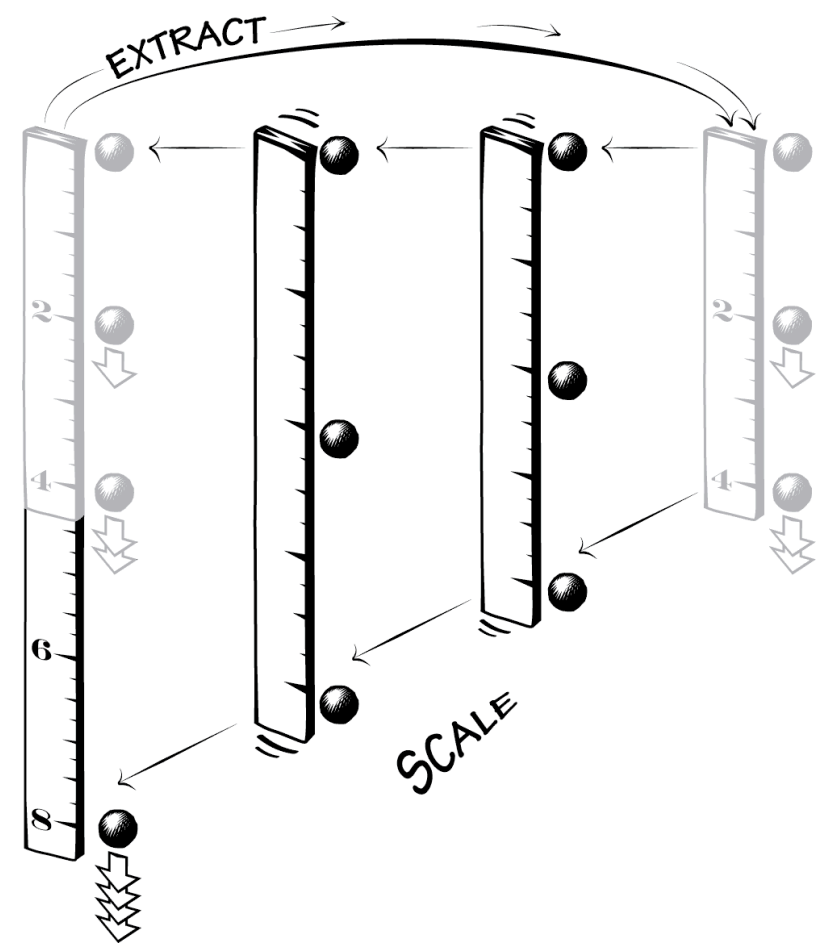

Figure 2. Original motion as a part of the scaled up motion.

\subsection{Downfall}

It is only in the third stage of his reasoning that Galileo stumbles. He now seeks to find the single time interval that satisfies the condition (ET). He continues:

but for the same moveable to pass the four braccia and the two in the same time cannot take place except in instantaneous [discontinuous] motion 
Here Galileo correctly notes one possible value for this unique time that the falling body requires to traverse four feet, eight feet and all other spaces. It is zero time, so that the motion is instantaneous; that is, it is a motion that covers a non-zero space in zero time. This value of zero time, with its associated instantaneous motion, is a degenerate solution to condition (ET) for motions governed by the velocity-distance law. The law is still satisfied but only through an awkward reading. If we double the space fallen, then the speed at the second position must be double the speed at the first. However the motions at both positions are instantaneous. We can conceive of the speed in the second motion as double that of the first in the sense that the second motion can cover twice the space as the first motion in the same zero time. We will not ponder it this issue further since Galileo does not indicate it as a problem.

There is another solution to (ET) that obtains in all non-degenerate cases of the speed-distance law. That is that the time to fall to four feet from rest or to fall eight feet from rest is, in both cases, infinite. This is a solution of (ET) that Galileo does not mention and, we believe, did not consider seriously. On first acquaintance, it may seem odd that the body would need an infinity of time to fall a finite space from rest under this law. Excepting the degeneracy noted, it is an unavoidable consequence of the speed-distance law of fall, as we will indicate below.

Galileo's failure to consider this infinite solution is the only flaw in his refutation of the speed-distance law. The failing was the tacit assumption that the law always leads to a finite time for a body to fall some distance from rest. Under that assumption, the only finite time that solves (ET) is zero time. So proceeding from that false assumption, Galileo concluded validly that instantaneous motion is the only motion possible under the speeddistance law. If, however, Galileo were to discard that false assumption, the nondegenerate motions could be restored, all of which require infinite time to fall any finite distance from rest and thus do solve (ET).

It is unfortunate that Galileo failed to consider these non-degenerate solutions since they would have completed what would otherwise have been a flawless refutation of the speed-distance law. Nevertheless, by arriving at (ET), Galileo has already correctly shown from the first two steps of his argument that the speed-distance law is not viable. 


\section{Did Galileo Really Intend this Argument?}

Is it really plausible that the argument just outlined is the one Galileo intended? We believe so and will try to make it more plausible by considering each of the steps of the argument.

\subsection{Evidence of Scaling}

Galileo's first step is to observe: 'When speeds have the same ratio as the spaces passed or to be passed, those spaces come to be passed in equal times'. A difficulty in interpreting this assertion is its brevity. Galileo does not specify to which motion or motions the speeds belong. Attending to that is key to interpreting this first step.

The interpretation most ready to hand is that the speeds belong to just one motion, that of fall under the speed-distance law, and that Galileo refers to ratios between different speeds at different spaces in this one motion. If that is all Galileo intended, the passage is not delivering what was promised in the immediately preceding sentence. That sentence asserts that the speed-distance law is 'as false and impossible as [it is] that motion should be made instantaneously, and here is very clear proof of it.' Under the one motion interpretation, Galileo's next step is just to declare in general terms the penultimate puzzling result, that the same time is needed for the motion to pass all spaces. The continuation of the sentence ${ }^{9}$ is then merely giving an instance of the general claim. Under this reading, Galileo's text is far from the 'very clear proof' promised; it is a blatant excise in circular reasoning. For the reader is not given a good reason to believe the general and troubling result that all spaces are passed in the same time. It is just asserted.

On our reading this awkwardness is escaped. The 'speeds' of Galileo's first step are associated with two motions. The second motion is a scaled up version of the first, in which speeds are scaled up in proportion to the spaces. There is no presumption that the motions obey the speed-distance law. The scaling result holds for any motions. The two

9 '... if therefore the speeds with which the falling body passed the space of four braccia were the doubles of the speeds with which it passed the first two braccia, as one space is double the other space, then the times of those passages are equal...' 
motions, Galileo reports, require the same time. On this reading, the conclusion is no longer a mere instance of the first premise.

So far there is nothing troubling. Galileo is merely reporting a benign fact concerning the scaling of motions. It is obviously correct for the case of the scaling up of a uniform motion. Then doubling the speed and doubling the distance will have exactly compensating effects on the time, so the time of passage will remain the same. Galileo knew this result. He states it for the case of uniform motion just a few pages before as his Proposition II, Theorem II ${ }^{10}$.

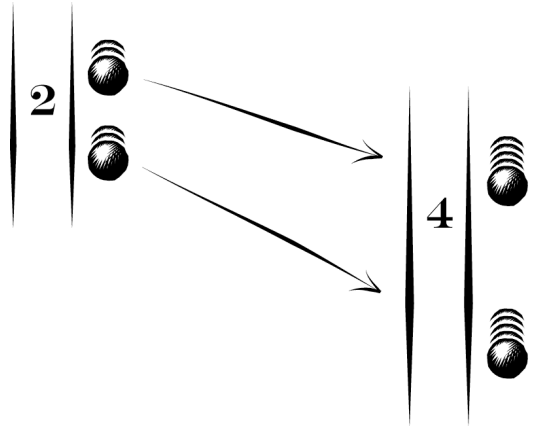

Figure 3. Scaling one part of the motion.

Galileo's present argument pertains to non-uniform motion. In this more general case, the scaling of speed and distance will still have exactly compensating effects, so the scaling result still holds. It is not hard to see that the extension is licit. Informally, consider just one small part of the space traversed in the motion prior to the scaling. Under the scaling by, let us say, a doubling of scale, that small part of the space will correspond to a small part of double the size in the rescaled space of the rescaled motion, as shown in Figure 4. Now the scaling also doubles the speed. As a result the time to traverse the small space in the original and scaled motion will stay the same. Finally, the original space can

10 'PROPOSITION II. THEOREM II. If a moveable passes through two spaces in equal times, these spaces will be to one another as the speeds. And if the spaces are as the speeds, the time will be equal' (Galilei, 1974, pp. 150). While the statement of the proposition does not mention a restriction to uniform motion, it is clear that this restriction was intended. The discussion comes from the section labeled 'Equable [Uniform] Motion' and, subsequently, Salviati closes the section with the remark, 'What we have just seen is all that our Author has written of equable [uniform] motion'. (Galilei, 1974, pp. 152). 
be decomposed exhaustively into many such small parts; and the rescaled space is decomposed into the same number of corresponding rescaled parts. In each case, the time to traverse the total space is the sum of the times to traverse their parts. Therefore the time to traverse the space before and after the scaling remains unchanged. (This informal argument can be made more precise using notions from the calculus, as shown in the appendix.)

Galileo, we maintain, was asserting the scaling result for the case of non-uniform motions. The extreme brevity of Galileo's statements leaves unclear whether he thought the result trivially obvious or hard won. Whichever, this result is certainly within his compass. Perhaps he could convince himself of it by an argument similar to the one just sketched. Perhaps he deemed the result so obvious that it could be asserted without further ado. Or perhaps he found that a proof was beyond the reach of his methods so he hoped his readers would accept his declaration of it. Many commentators have noted the awkwardness of Galileo's proof a few pages later of the mean speed theorem that involves the somewhat dubious summing of infinitely many lines.

Whatever may have been behind the brevity of Galileo's presentation, we cannot doubt that this sort of scaling result was one for which Galileo demonstrated great facility. The analysis of laws of fall in the Third Day of the Two New Sciences is followed a few pages later in the Fourth Day with a number of scaling arguments for non-uniform motions on inclined planes ${ }^{11}$. The analysis is moreover preceded by an extended treatment of the strength of structural members in the Second Day, and before that with a discussion of the scaling of boats on the very first page of the First Day. The treatment of the strength of structural members was concerned centrally with how the strength of these members varied as they were scaled up in size. Galileo's analysis was carried out for regular shapes like prisms and cylinders. However Galileo showed no hesitation ${ }^{12}$ in applying these result to very much less regular shapes. Immediately following his treatment of the strength of cylinders under scaling, he declared results applying to the very irregular shapes of bones.

11 Of particular interest is Proposition VI Theorem VI of the Fourth Day, in which the final distances and speeds are scaled in equal proportion, and the resulting motions shown to occur in equal times.

12 For example, see (Galilei, 1974, pp. 126-128). 
Figure 5 is an illustration from the Two New Sciences $^{13}$. If irregular figures like this did not daunt him when it came to scaling, presumably neither would non-uniform motions.

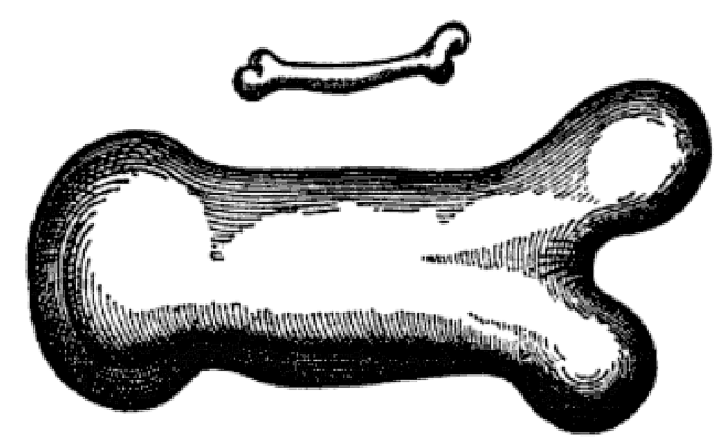

Figure 4. Scaling Bones in Galileo's Two New Sciences.

What we learn from this analysis in the Second Day is that Galileo was accustomed to thinking in terms of scaling, that he was quite adept at scaling arguments and that he was quite willing to extend scaling results from regular to irregular figures. Therefore it seems quite reasonable that Galileo would be comfortable thinking of the speed-distance law in terms of scaling arguments and that he would take the scaling result for constant motion that he had stated in his Proposition II. Theorem II and extend it to non-uniform motion.

\subsection{Getting to Galileo's Conclusion}

Galileo's opening remark, 'When speeds have the same ratio as the spaces passed or to be passed, those spaces come to be passed in equal times,' refers to two general motions, one scaled into the other. The remark then continues to note that the scaling relation holds within the one more specific motion of fall governed by the speed-distance law:

...if therefore the speeds with which the falling body passed the space of

13 Public domain image from (Galilei, 1914, pp. 131). 
four braccia were the doubles of the speeds with which it passed the first two braccia, as one space is double the other space,...

Therefore the scaling result applies through self-similarity to the motion and he can now conclude: ' ... then the times of those passages are equal...'

This identification of self-similarity of Step 2 and its outcome (ET) follows without need for further comment. What does bear comment is the concern that Galileo's conception of speed does not coincide exactly with our modern notion. Our analysis does not require it to coincide, but only that it agree enough to sustain the inferences we report, as we believe it does. For example, the idea of a speed-distance law of fall and that it is a self-similar motion requires only that the magnitude, speed, be representable geometrically in Galileo's standard manner. Galileo routinely depicted the speed-time law of fall with the figure of a triangle ${ }^{14}$. A similar representation is possible for the speed-distance law. Such a diagram is shown in Figure 5. In such a diagram, one side of the triangle represents the distance fallen $\mathrm{OA}, \mathrm{OB}, \mathrm{OC}, \ldots$ and another side the speed at corresponding instants, $\mathrm{OA}^{\prime}$, $\mathrm{OB}^{\prime}, \mathrm{OC}^{\prime}, \ldots$ :

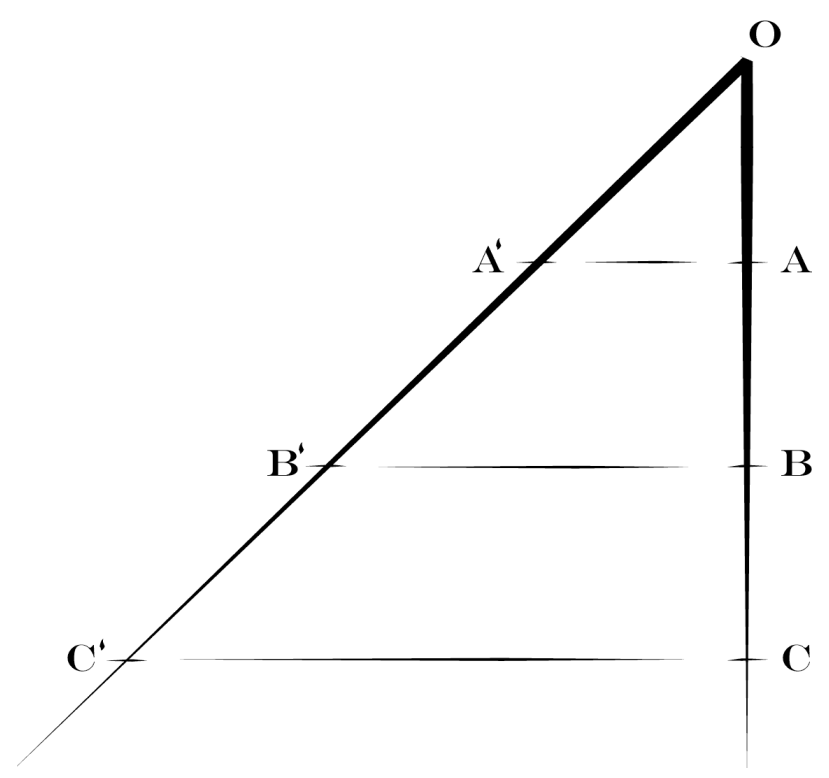

Figure 5. A Galilean representation of the speed-distance law.

14 For an overview of Galileo's standard triangular depiction of speed and distance, as well as a comparison of his concept of speed to the modern notion, see (Damerow et al., 2002). 
The speed-distance law is expressed in the similarity of the triangles $\mathrm{OAA}^{\prime}, \mathrm{OBB}^{\prime}, \mathrm{OCC}^{\prime}$... If the distance fallen $\mathrm{OB}$ is twice the distance $\mathrm{OA}$, the similarity of triangles $\mathrm{OAA}^{\prime}$ and OBB' assures us that the speed gained, $\mathrm{OB}^{\prime}$, is twice the speed $\mathrm{OA}^{\prime}$, at the corresponding moments. The self similarity of the motion is expressed in the fact that the triangle $\mathrm{OCC}^{\prime}$ is similar to triangles that represent only parts of the motion, OAA' and OBB'.

Finally, further comment is needed on the failure in Step 3 of Galileo's argument in which he overlooks that non-degenerate forms of the speed-distance law lead to infinite times of fall from rest. That he did neglect it can be affirmed because of discussion elsewhere in the Third Day. Why he might neglect this possibility may be that he was working with a particular conservative notion of infinity.

Shortly after Galileo's refutation of the speed-distance law is laid out, Simplicio explains some concerns relating to the infinite variability of speed in laws of fall. His concern is expressed in the context of a reversal of a motion of fall, a rising body that comes to rest. If the rising body must pass through an unlimited number of different degrees of speed to come to rest, Simplicio asserts that the body will continue moving indefinitely and never come to rest.

Galileo responds in the voice of Salviati:

This would be so, Simplicio, if the moveable were to hold itself for any time in each degree; but it merely passes there, without remaining beyond an instant. And since in any finite time [tempo quanto], however small, there are infinitely many instants, there are enough to correspond to the infinitely many degrees of diminished speed. (Galilei, 1974, pp. 157.)

We need not ponder just yet how Galileo's argument here works. For present purposes, the essential point is that Galileo discounts the possibility of a law of fall requiring infinite time for the rising body to come to rest. That is equivalent to asserting that a falling body always requires a finite time to fall a finite distance from rest. Therefore we know that, had Galileo considered the possibility that infinite time solves the condition (ET), he would have discounted it as inapplicable to the motion of fall. 
The reason why Galileo discounted this possibility may have something to do with his concept of infinity. One must distinguish Galileo's use of infinity to count a number of objects from his use of infinity to quantify a continuous physical magnitude, like force or speed. In discussions of the former, Galileo is perfectly happy to talk about an actual infinity of objects ${ }^{15}$. But in discussions of the latter, Galileo seems to restrict himself to some kind of potential infinity. For example, he imagines (through Salviati) that one might 'increase in infinitum the force applied' or that 'speed may be increased or diminished in infinitum' (Galilei, 1974, pp. 132 and pp. 156). Galileo's use of words like 'increase' and 'decrease' in these cases suggests an unwillingness to consider the possibility that continuous physical magnitudes might actually be infinite. Galileo's failure to consider an infinite time solution may thus be due to this conservative notion of infinity.

It is interesting, in addition, to examine Galileo's argument for the finitude of times of fall, for the argument is fallacious. The fallacy can be seen in Galileo's terms. To see it this way, consider some body falling from rest under the speed-distance law. We can divide the space through which it falls without limit into spaces that are related in geometric ratio. That is, they are bounded by the positions

$$
\ldots, 1 / 16,1 / 8,1 / 4,1 / 2,1,2,4,8, \ldots \text { feet }
$$

Now recall the Galileo's self-similarity result. The motion over the space from $1 / 4$ to $1 / 2$ foot scales up by a factor of 4 to the motion over the space from 1 to 2 feet; therefore the two spaces are passed in the same time. Repeatedly applying this result, we infer that the time to space the space from 1 to 2 feet is the same as required for $1 / 2$ to 1 feet; and for $1 / 4$ to $1 / 2$ foot; and for $1 / 8$ to $1 / 4$ foot; and so on indefinitely. In order to reach any finite position past the initial zero position, the body must fall through an unlimited number of these spaces, each requiring the same time for passage. Therefore the body will require unlimited time to fall to any finite position. (If a motion of this type is discomforting, see the Appendix for further discussion.)

15 For example, in the discussion of Aristotle's 'wheel paradox,' Salviati argues that a rolling wheel may cross 'infinitely many voids' as it rolls along (Galilei, 1974, pp. 38). More famously, Galileo later points out that the 'square numbers are as numerous as all the numbers', both infinite (Galilei, 1974, pp. 40). 


\section{Conclusion}

It has long been recognized by commentators that the speed-distance law of fall yields an exponential dependence of distance on time that does not admit the case of a body falling from an initial state of rest. Indeed the result can be made evident to anyone who has the barest familiarity with the differential calculus. It arises as the solution to a simple differential equation, which sets the rate of change of distance proportional to distance. For this reason, it is now evident to everyone that the speed-distance law fails as a law of fall.

What we urge in this note is that Galileo essentially discovered this good reason to dismiss the law. Unlike modern commentators, he did not have the methods of differential calculus to call upon. He had to explore he properties of motions, such as those prescribed by the speed distance law, using the methods of geometry. His geometrical approach all but succeeded. By using a combination of a scaling and self-similarity arguments, Galileo identified the property of its motion that dismiss it as a candidate law of fall: a body requires the same (infinite) time to fall from rest to any nominated distance.

Galileo's geometrical arguments identified this key disqualifying property. But he failed to realize that the one fixed time taken to fall to any nominated distance is infinite. In the last step of the analysis, he confused this case with the one other that shares this property, instantaneous motion, in which a falling body takes no time to pass all finite distances.

\section{Appendix}

\section{A.1 Scaling}

We can readily see, using more modern methods, that Galileo's scaling result for motion is correct. To see it, let a body move through a distance $L$ with coordinates $x$ extending from $x=0$ to $x=L$ such that it has speed $v(x)$ at each value of $x$. We may use any well-behaved function for $v(x)$. ('Well-behaved' here just means that the inverse speed is integrable.) We consider a second motion in which both distances and speeds are scaled 
up by a factor of 2 . This motion covers the interval $x^{\prime}=0$ to $x^{\prime}=2 L$ with the velocity $v^{\prime}\left(x^{\prime}\right)$, where this function is specified by the condition $v^{\prime}\left(x^{\prime}\right)=v^{\prime}(2 x)=2 \cdot v(x)$.

The scaling result is that the two motions will require the same time. The quickest way to see it uses infinitesimal argumentation. The time required to cover some small interval $x$ to $x+\mathrm{d} x$ is $\mathrm{d} t=\mathrm{d} x / v$. This small interval of space scaled up is the interval $x^{\prime}=2 x$ to $x^{\prime}+\mathrm{d} x^{\prime}=2 x+2 \mathrm{~d} x$, where the velocity is $v^{\prime}\left(x^{\prime}\right)=v^{\prime}(2 x)=2 \cdot v(x)$. The time to traverse it is just $\mathrm{d} t^{\prime}=\mathrm{d} x^{\prime} / v^{\prime}=(2 \cdot \mathrm{d} x) /(2 v)=\mathrm{dt}$. Since the distance $L$ of the unscaled motion and the distance $L^{\prime}=2 L$ of the scaled motion can be decomposed into infinitely many paired, small intervals, each requiring the same time $\mathrm{d} t^{\prime}=\mathrm{d} t$, it follows that both motions require the same time overall.

A more precise rendering of this analysis employs integral calculus. The time $T^{\prime}$ for the scaled motion to traverse the distance $L^{\prime}=2 L$ is given by

$$
T^{\prime}=\int_{0}^{L^{\prime}} \frac{d x^{\prime}}{v^{\prime}\left(x^{\prime}\right)}=\int_{0}^{2 L} \frac{d(2 x)}{2 \cdot v(x)}=\int_{0}^{L} \frac{d x}{v(x)}=T .
$$

As the computation shows, $T^{\prime}$ equals the time $T$ required for the unscaled motion.

There is also a graphical way to see this same result. The time required for some motion is just the area under the curve arising when we plot the inverse of speed against distance. Figure 6 shows a plot of inverse speed against distance for some motion that passes from $x=0$ to $x=L$. It also shows the scaled motion that now covers double the distance from $x=0$ to $x=2 L$, but at twice the speed. This doubling of the speed has the effect of halving the inverse speed. As a result the area associated with the scaled motion is the same as the area associated with the unscaled motion. That is, the traversal times are the same. 


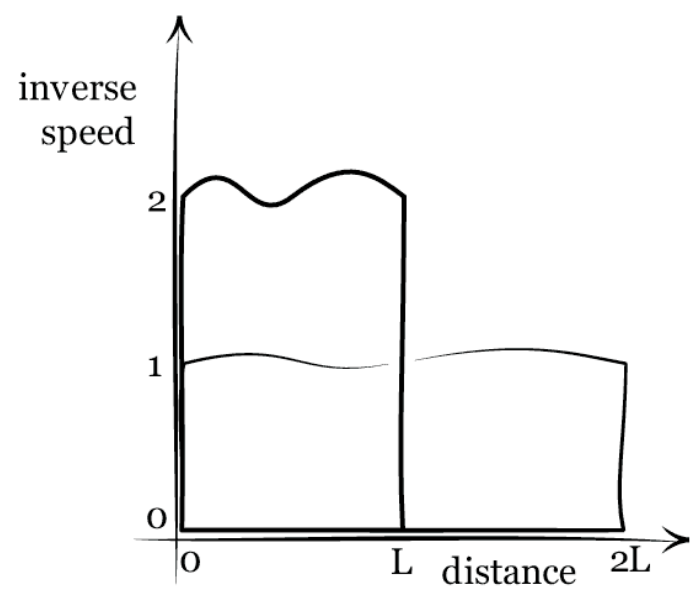

Figure 6. Original and scaled motions require the same time.

\section{A.2 Infinite Time of Fall}

That the speed-distance law requires a body to fall for an infinite time from rest to achieve any finite distance is most easily seen with a little calculus. For simplicity, let us take the case of speed $v=\mathrm{d} x / \mathrm{d} t$ numerically equal to distance $x$. Then the fall is governed by the differential equation $\mathrm{d} x / \mathrm{d} t=x$. This equation has the familiar solution

$$
\frac{x}{x_{1}}=\exp \left(t-t_{1}\right)
$$

It tells us that, if a body has arrived as position $x_{1}$ at time $t_{1}$, then, if it continues to fall for an additional time $t-t_{1}$, it will have arrived at position $x$. If we select $x_{1}=0$ as our initial point, then the ratio $x / x_{1}$ diverges. It follows immediately that the time $t-t_{1}$ needed to fall to any finite position $x>0$ is infinite.

Alternatively, one can invert the above relation between distance fallen $x$ and the time required $t$ to recover the relation $t=\log x$. The motion that arrives at $x>0$ started at position $x=0$ at time $\log 0$, which is negative infinity. Therefore to arrive at $x>0$, the body has been falling for infinite time.

This same result can be see graphically if we plot inverse speed against distance. Figure 7 below on the left shows a plot of speed versus distance for the case of motion in which speed $=$ distance over the distance $x=0$ to $x=4$; it also shows the scaled motion over 
$x=0$ to $x=8$. The two curves coincide in the region $x=0$ to $x=4$ because of the self-similarity. The figure on the right shows the corresponding motions with inverse speed plotted against distance.
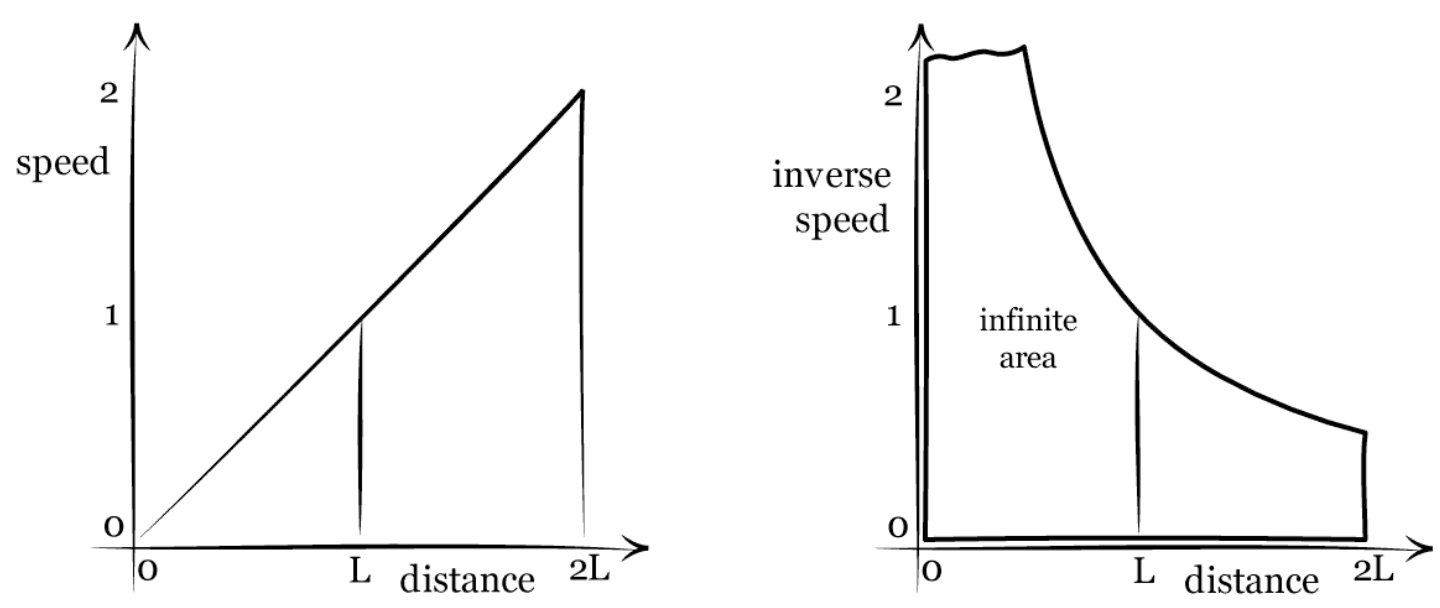

Figure 7.

The inverse speed curve is an hyperbola and the area under it is infinite; that entails that the time required to cover the distance is infinite. The area under the curve between $x=0$ and $x=4$ is the same as the area under the curve between $x=0$ and $x=8$; they are both infinite. As a result, this motion satisfies the condition (ET).

There is, of course, a metaphorical element in this talk of 'falling for an infinite time.' More precisely what this locution indicates is that the body has been falling for all times in the past. At any finite time in the past, the mass was already underway in its fall; there is no finite time in the past at which it was at rest. Since all times in our history are at finite times in the past, there is no moment in time at which the mass was at rest. It has always been moving. The idea of its rest an infinite time ago merely arises in a limit process that describes a state never actualized.

Finally, if the idea of a motion that requires infinite time to be completed is troubling, it might be helpful to note that just such a motion can arise in a Newtonian model. That model consists of a perfectly frictionless hemisphere in a gravitational field 
over which a point mass slides ${ }^{16}$. The point mass is projected up the hemisphere with exactly the initial velocity need to have it come to rest at the hemisphere's apex. A short calculation shows that this motion will require an infinity of time be completed. Because of the time reversibility of Newtonian theory, the rise of the mass to rest at the apex can be reversed in time to yield a falling motion admitted by the theory. If we conceive this reversed motion as starting at the apex, it must have been underway for infinite time for it to have arrived at any position away from the apex. The law of fall governing the mass in the surface of the hemisphere corresponds to the speed-distance law in the vicinity of the apex, in so far as we can use the approximation that $\sin \mathrm{A}$ equals $\mathrm{A}$, for small angles $\mathrm{A}$.

\section{References}

Cohen, I. B. (1956) Galileo's rejection of the possibility of velocity changing uniformly with respect to distance, Isis, 47(3), 231-235.

Damerow, P., McLaughlin, P., Freudenthal, G., and Renn, J. (1992) Exploring the Limits of Preclassical Mechanics. (Springer-Verlag New York, Inc.)

Drake, S. (1969) Galileo's 1604 Fragment on Falling Bodies (Galileo Gleanings XVIII), The British Journal for the History of Science, 4(4), 340-358.

- (1970) Uniform Acceleration, Space, and Time (Galileo Gleanings XIX). The British Journal for the History of Science, 5(1), 21-43.

Fermat, P. (1894) Fermat a Gassendi (1646?), in: Tannery and Henry (eds.) Oeuvres de Fermat, volume 2 (Paris: Gauthier-Villars et Fils), 267-276.

Galilei, G. (1914) Dialogues Concerning Two New Sciences [Trans. Crew, H. and de Salvio, A.] (MacMillan).

Press).

(1974) Two New Sciences [Trans. Drake, S.] (Madison: University of Wisconsin

Galluzzi, P. (2001) Gassendi and l'affaire Galilée of the laws of motion, in: J. Renn (ed.) Galileo in Context (New York: Cambridge University Press) 239-275.

Hall, A. R. (1958) Notes and correspondence: Galileo's fallacy, Isis, 49(3), 342-349.

Mach, E. (1919) The Science of Mechanics: A Critical and Historical Account of its Development. [Trans. McCormack, T. J.] (Chicago, London: The Open Court Publishing Co.).

16 To preclude confusion, this Newtonian example is not the pathological case of the indeterministic dome of (Norton, 2008). 
Mersenne, M. 1973a. Les nouvelles pensées de Galilée, mathématicien et ingénieur, Volume 1, critical edition, [Eds. Costabel, P. and Lerner, M.-P.] (Paris: J. Vrin).

. 1973b. Les nouvelles pensées de Galilée, mathématicien et ingénieur, Volume 2, critical edition, [Eds. Costabel, P. and Lerner, M.-P.] (Paris: J. Vrin).

Norton, J. D. (2008) The dome: An unexpectedly simple failure of determinism, Philosophy of Science, 75(5), 786-798.

Palmieri, P. (2009) Radical mathematical Thomism: beings of reason and divine decrees in Torricelli's philosophy of mathematics, Studies in History and Philosophy of Science, 40(2), 131-142.

Roberts, B. W. (Forthcoming) How Galileo dropped the ball and Fermat picked it up, Synthese (DOI 10.1007/s11229-009-9705-7).

Tenneur, J.-A. (1649) De motu naturaliter accelerato tractatus physico-mathematicus, in quo discussis, aliquot recentiorum sententiis, vera accelerationis gravium ratio demonstratur (Paris). 